\title{
Is there a search in fixed-set memory search?
}

\author{
MICHAEL A. STADLER \\ Purdue University, West Lafayette, Indiana \\ and \\ GORDON D. LOGAN \\ University of Illinois, Champaign, Illinois
}

\begin{abstract}
We discuss the hypothesis that structural factors in the fixed-set memory-search task may contribute to the standard linear set-size effect typically observed in this task. We constructed a lexical decision task that contained the same structural factors as the memory-search task. Two structural factors are discussed: (1) the priming of the probe by the memory set, and (2) the confounding of repetitions per item with set size. Our experiment demonstrated that these structural factors play a role in the set-size effect. The implications of these effects for the memory. search task and for models proposed to account for memory-search data are discussed.
\end{abstract}

One of the most well-known tasks in modern psychology is the memory-search task popularized by Sternberg $(1966,1967,1969,1975)$. In this task, a subject is asked to compare an item, or probe, against a previously memorized list of items, called the memory set, and to decide whether or not the probe item is a member of the memory set. A common finding is that reaction time for the decision on the probe item is a linear function of the number of items in the memory set, both for items that are members of the set (positive probes) and for items that are not (negative probes). Sternberg interpreted these data to indicate a serial and exhaustive comparison of the probe item to the items in the memory set: serial because the functions are linear, suggesting a one-by-one comparison process, and exhaustive because the positive and negative functions are parallel, suggesting that, in both cases, the subject searches the entire memory set.

Since the introduction of the memory-search task, it has been used widely and in many variations, and many alternatives to Sternberg's interpretations have followed (see Townsend \& Ashby, 1983, for a thorough discussion of these). The central theme in this literature has been the idea that the increases seen in reaction time with the addition of items to the memory set (the set-size effect) reflect the operation of some sort of comparison process in which the probe is compared to the items in the memory set.

However, there are structural factors in the memorysearch procedure that may account, at least in part, for the set-size effect without assuming a comparison process. In the fixed-set version of the memory-search task, memory set size is manipulated between blocks; subjects

This research was supported by Grant BNS 8510365 from the National Science Foundation to Gordon Logan. The authors would like to thank Michael Corballis, Alice F. Healy, James H. Neely, Henry L. Roediger III, and anonymous reviewers for their comments on earlier drafts of this paper. Requests for reprints may be addressed to Michael Stadler, who is now at the Department of Psychology, Louisiana State University, Baton Rouge, LA 70803-5501. use one memory set for several trials in succession. This is in contrast to the varied-set procedure, in which subjects are given a new memory set on each trial before presentation of the probe. In the fixed-set procedure, memory set size is typically confounded with the number of trials per item (e.g., Briggs \& Johnsen, 1973; Burrows \& Murdock, 1969; Graboi, 1971; Kristofferson, 1972; Lively, 1972; Logan, 1978; Neisser, Novick, \& Lazar, 1963; Ross, 1970; Simpson, 1972). Because of this confounding, the probability of an immediate repetition decreases as set size increases. For example, in the present experiment, the probability of an immediate repetition is $0.5,0.25,0.16$, and 0.125 , respectively, for Set Sizes 1-4. It has been shown (e.g., Kirby, 1980; Komblum, 1969, 1973; Rabbitt \& Vyas, 1973) that when one stimulus event in a choice reaction-time task is repeated on two consecutive trials, reaction time to the second presentation is typically shorter than reaction time to the first. Thus, there should be less reaction-time benefit from stimulus repetitions as set size increases, mimicking the set-size effect that would be produced by a comparison process.

Another structural factor may be the dilution of a priming effect. It is commonly assumed that an item's representation is primed (or its memory strength increased) when the item is presented as a member of the memory set or when it appears as a probe (see, e.g., Baddeley \& Ecob, 1973). When there is only one item in the set, it receives the maximum amount of priming. When the memory set is larger than a single item, priming is diluted, or shared, across all the items in the memory set. This notion is similar to that of the dilution of the Stroop effect (Kahneman \& Chajzyck, 1983), in which the Stroop effect is diminished by the presence of irrelevant words in a display. When a probe from the memory set is presented, the reaction time to the probe may depend in part on the amount of priming the probe item received when it was presented in the memory set, which will be smaller the larger the memory set. In addition, because 
the number of different probes increases as memory set size increases, any priming that occurs when an item appears as a probe will be diluted more as the memory set size increases. Thus, a set-size effect may occur, but not necessarily or completely because of a comparison process (see also Stadler, 1989).

The logic of the experiment reported below was to construct another task that would not require a comparison process, but would contain most of the same structural properties as the fixed-set memory-search task. If performance in the second task was similar to that in the memory-search task, then the structural properties shared by the two tasks would be the most reasonable explanation. One such task is the lexical decision task, in which the subject is asked to decide if a string of letters is a word or not. This task presumably relies on access to long-term memory, but not on a short-term memory-search process of the sort used to explain memory-search phenomena. We constructed a lexical decision task that would have the same repetition properties as a fixed-set memorysearch task. The lexical decision task also shared most of the same dilution of priming structure, in that items in the lexical decision task were presented as probes the same number of times as were items in the memory-search task. However, because it was not necessary for the lexical decision task, and in order to avoid inducing a memory-search strategy in the lexical decision subjects, the positive (memory) set was not presented to the lexical decision subjects at the beginning of a block of trials. Thus, any dilution of priming from the initial presentation of the positive set or from its rehearsal would not occur in the lexical decision condition.

\section{METHOD}

\section{Subjects}

The subjects were 64 students enrolled in introductory psychology classes at Purdue University. Half of the subjects performed the memory-search task and half performed the lexical decision task. All of the subjects reported English as their native language and had normal or corrected vision.

\section{Apparatus and Stimuli}

The experiment was controlled by a PDP 11/03 computer, which displayed the stimuli on a Tektronix Model 604 point-plot CRT. Letters that made up the stimuli were formed by illuminating approximately 20 points in a $5 \times 7$ dot matrix, and subtended about $0.43^{\circ} \times 0.57^{\circ}$ of visual angle from a distance of $60 \mathrm{~cm}$. The subjects used a headrest to maintain this distance. They responded by pressing one of the two outer telegraph keys on an eight-key panel.

Sixteen blocks of 48 trials were prepared for each subject, four blocks each for Set Sizes 1-4. The stimuli were drawn randomly from a pool of 340 five-letter word-nonword pairs. The words were five-letter nouns from the Kucera and Francis (1967) word norms and ranged in frequency from a low of 8 per million to a high of 787 per million, with a mean of 75 per million. The nonwords were constructed by changing one letter of each of the 340 words in the pool and were pronounceable.

For each subject, 20 word-nonword pairs were selected to be used as the positive and negative items. In the memory-search task, the 20 words from the pairs were used, 10 as positive items and
10 as negative items. In the lexical decision task, the words in 10 of the pairs of items were used as positive items and the nonwords from the other 10 pairs were used as negative items. A given word and the nonword derived from that word were never used for the same subject. The items were randomly assigned to set size and status as positive or negative without replacement from the 20 items. Thus, each item appeared at only one set size and as only a positive or a negative item. In each block of 48 trials, half required positive, or "yes," responses and half required negative, or "no," responses. Each word from the positive and negative lists for each block appeared as the probe item an equal number of times. Thus, each item appeared a total of $96,48,32$, and 24 times for set sizes of $1,2,3$, and 4 , respectively.

\section{Procedure}

A trial began with the presentation of a fixation point and a tone for $500 \mathrm{msec}$. The fixation point was immediately replaced by the item to which the subjects were to respond. This item also appeared for $500 \mathrm{msec}$. The subjects were given up to $2 \mathrm{sec}$ after stimulus onset to make their response. At the end of this period, the fixation point for the next trial appeared. The subjects in the memory-search group were instructed to respond to each stimulus by pressing the "yes" button if the word was a member of the memory set they had been given, the "no" button if it was not. The subjects in the lexical decision group were instructed to press "yes" if the stimulus was a word and "no" if it was a nonword.

Sessions for both groups began with instructions that emphasized the importance of responding quickly while maintaining about $90 \%$ accuracy. The subjects in the memory-search group were told the memory set for each block before the experimenter began the presentation; the subjects in the lexical decision group were told that words and nonwords would appear but were not informed about the specific items that would appear in the block. Half of the subjects in each group were told to respond "yes" by pressing the left key with their left index finger and "no" by pressing the right key with their right index finger; the other half were given the opposite instruction. Blocks 1,2 and 3, 4 for each set size were run consecutively with a short break between Blocks 2 and 3; order of presentation of the four set sizes was counterbalanced across subjects.

\section{RESULTS}

Both reaction-time and error data were recorded, but only the reaction-time analyses will be presented because error rates were so low. The error rates are presented in Table 1 and the reaction-time data are displayed in Figure 1. The $p$ value employed in all analyses was .05 .

The effects of set size and response type for the two tasks are displayed in Figure 1. As is evident in the figure,

Table 1

Mean ( $\pm S E M$ ) Percent Correct Responses by Condition and Set Size

\begin{tabular}{lcccc}
\hline & \multicolumn{5}{c}{ Set Size } \\
\cline { 2 - 5 } Condition & 1 & 2 & 3 & 4 \\
\hline \multicolumn{5}{c}{ Memory Search Task } \\
Positive & $98 \pm .60$ & $96 \pm .48$ & $95 \pm .43$ & $95 \pm .39$ \\
Negative & $97 \pm .58$ & $96 \pm .31$ & $96 \pm .37$ & $96 \pm .22$ \\
\multicolumn{5}{c}{ Lexical Decision Task } \\
Positive & $96 \pm .72$ & $96 \pm .49$ & $97 \pm .35$ & $97 \pm .31$ \\
Negative & $98 \pm .50$ & $94 \pm .44$ & $95 \pm .48$ & $94 \pm .50$ \\
\hline
\end{tabular}




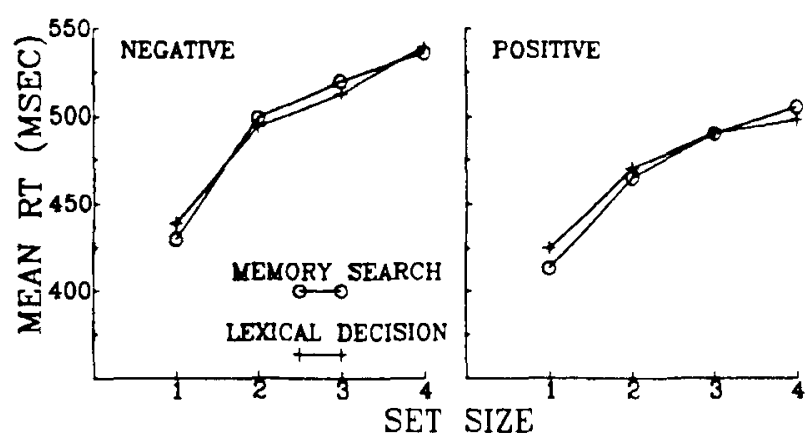

Figure 1. Mean reaction time for negative and positive responses as a function of task and set size.

the data from the two tasks are quite similar. The grand mean reaction times were $483 \mathrm{msec}$ for the memorysearch task and $484 \mathrm{msec}$ for the lexical decision task. In both tasks, as the number of items in the positive and negative sets increased, so did mean reaction time. Negative responses took slightly longer than positive responses, and the differences between the types of responses was slightly larger for the largest set sizes. A task (memory search vs. lexical decision) $\times$ set size (one through four) $X$ response type (positive vs. negative) analysis of variance with subjects nested in task was used to examine these effects. The task factor was not significant, nor were any interactions of this factor and the others (all $F \mathrm{~s}<1$ ). Further analyses were performed separately for the two tasks to allow comparison of the different effects and their magnitudes between the two tasks. The statistics for both tasks will be presented together; the memory-search statistics will precede the lexical decision statistics.

Since the effect of set size is generally linear, a trend analysis was performed for each task to test the linearity of the function. The linear trend was significant for both tasks $\left[F(1,93)=168.30\right.$ and $108.42, M S_{e}=15,646.29$ and $16,205.99]$. Deviations from linearity were also significant $[F(2,93)=12.22$ and 6.76]. These significant deviations are most likely due to the influence of the points at Set Size 1, which often depart from the trend apparent in larger set sizes (Sternberg, 1975). When these data were not included in the analysis, the deviations from linearity were no longer significant (both $F \mathrm{~s}<1, M S_{\mathrm{e}}=14,221.74$ and 16,749.36), but the basic trends in the data and our interpretations do not change. Because of this, the data from Set Size 1 were not excluded from further analyses. The main effect of response type was significant $\left[F(1,31)=83.68\right.$ and $44.74, M S_{\mathrm{e}}=3,977.58$ and $7,872.55]$. As was observed above, the slopes of the positive and negative functions are slightly different (30 and 24 for the positive functions and 34 and 31 for the negative functions); this produced significant set size $\times$ response type interactions $\left[F(3,93)=4.25\right.$ and $5.26, M S_{\mathrm{e}}=$ 745.16 and 968.07$]$.

Structural factors may produce comparable set-size effects when performance is averaged over all trials, but they should not produce comparable effects very early in practice. By definition, repetition cannot affect performance on the first presentation of items in either task; the contribution of repetition to the set-size effect should emerge as practice progresses. Thus, data from the first presentation provide an estimate of performance that is uncontaminated by repetition effects, and subsequent data should reveal the confounding effects. To examine this aspect of the data, we averaged reaction times over items for each presentation in each set size (there was one item per repetition in Set Size 1, two items in Set Size 2, etc.) and calculated the slopes of linear regressions for each presentation. The mean slopes for the first 12 presentations are presented in Figure 2. ${ }^{1}$ Because so few observations were available from which to estimate the slope of each presentation for each subject, the data were averaged over response type.

There are clear differences in the slopes across presentations and tasks. In both tasks, the slope was small on the first presentation (16.7 and $-4.7 \mathrm{msec} /$ item in memory search and lexical decision, respectively) but increased substantially on the second presentation. This difference reflects the contribution of repetition to the set-size effect. When repetition could not influence performance (the first presentation), the slope of the set-size effect was very small. The effects of repetition are then observed as early as the first repetition (the second presentation). The slopes of the two tasks appear to differ at early presentations, but not so much at later ones. This difference may reflect the influence of other processes in the memory-search task. Some processes other than repetition-a memory scan or dilution of priming, for example-might contribute to the set-size effect on the first presentation.

A 2 (task) $\times 12$ (presentation) analysis of variance with subjects nested in task largely confirmed these observations. The effect of presentation was significant $\left[F(1,11)=4.35, M S_{e}=3,524.51\right]$, as was the effect of task $\left[F(1,11)=4.42, M S_{\mathrm{e}}=18,683.52\right]$. However, presentation did not interact with task $\left[F(1,11)=1.28, M S_{\mathrm{e}}=\right.$ $1,037.58]$. Planned contrasts between consecutive presentations within each task indicated that Presentation 1 was significantly different from Presentation 2, but no subsequent pairs of presentations differed reliably. Planned contrasts between tasks at each presentation revealed significant differences at Presentations 1, 2, 3, and 6. All significant comparisons exceeded $t(31)=1.697$, and the $S E$ of all contrasts was 8.05 .

Our interpretation of the data presented above assumes that the subjects in the lexical decision condition did not search short-term memory in the same way as the subjects in the memory-search condition might have. An alternative interpretation of our data may be that the subjects in the lexical decision group induced the positive set and performed the lexical decision task as if it were a memory search, responding "yes" if the probe word was in the positive set and "no" if it was not. However, there is evidence against such an alternative explanation. The slope in the lexical decision task increased dramatically from the first presentation to the second. This occurred 


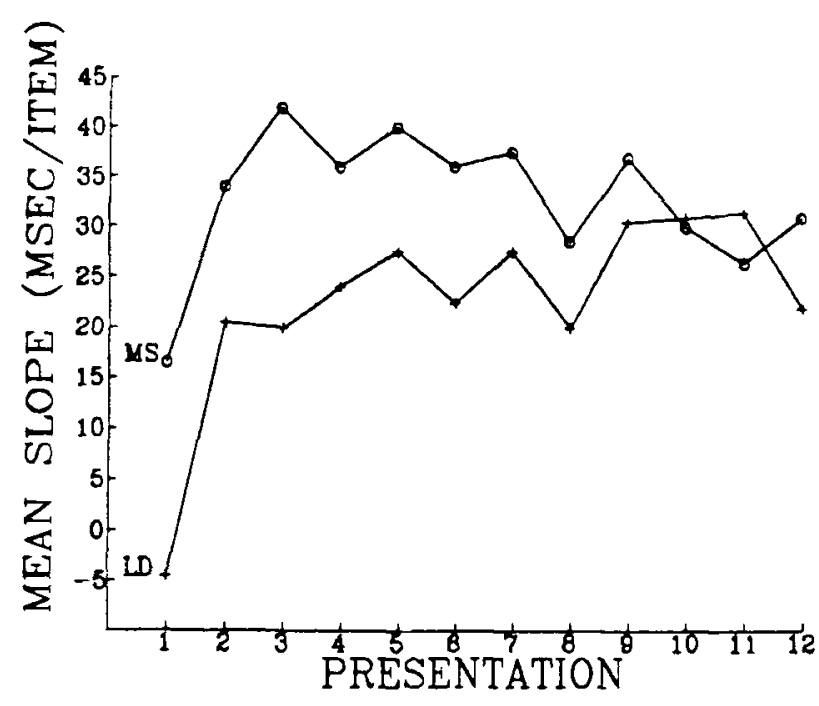

Figure 2. Mean slope of the set-size function in each task for Presentations 1-12. MS = memory search, and LD = lexical decision.

too soon for the subjects to have confidently induced the memory set because the second presentation of one item might have occurred before the first presentation of other items (due to random presentation order). ${ }^{2}$ However, the second presentation is not too early for effects of repetition to appear. The effects of repetition can also be seen in the memory-search task, in which the slope also increases from the first to the second presentation. If our results were attributable solely to the induction of a memory-search strategy by the subjects in the lexical decision condition, such an effect would not be expected.

We collected other evidence that suggests that the subjects in the lexical decision condition did not induce the memory-search strategy. The possibility that they might induce this strategy occurred to us midway through the experiment, so we asked the remaining subjects to recall all of the items from all of the positive sets (recall all the words for the lexical decision subjects). The subjects in the memory-search group recalled 7.5 out of the 10 words on the average, whereas the subjects in the lexical decision group recalled only 3.6 out of the 10 words $[t(14)=4.592, S E=0.8439]$. The lexical decision subjects clearly did not process the words in the same way as did the memory-search subjects. In other words, the short-term memory processes (e.g., rehearsal) employed by the memory-search subjects to maintain the memory set presumably led to the long-term storage of an average of 7.5 of the items. The lexical decision subjects clearly did not engage the same short-term memory processes; their recall was much poorer.

Sternberg (1969) has argued that the fixed-set procedure involves representation of the memory set in both short-term and long-term memory, but that the short-term representation, which he argued is maintained by rehearsal, is the one that is scanned. Of course, rehearsal is one process by which information is assumed to be transferred from short-term to long-term memory. This interpretation suggests that the subjects who performed the lexical decision task did not engage in rehearsal to the same degree as the subjects in the memory-search condition. However, we need not make assumptions about exactly what short-term memory processes were employed by the subjects. The difference in recall scores suggests that whatever processes the subjects employed in memory search to maintain the memory set were not employed in lexical decision. Given the repetition data and the recall data, we conclude that it is unlikely that the subjects in the lexical decision condition induced a memory-search strategy.

\section{DISCUSSION}

Our aim was to determine whether structural factors in the memory-search procedure contribute to the set-size effect. Our experiment produced set-size effects in a lexical decision task where one would otherwise not expect to find them. Below, we address some implications of these findings, along with some potential qualifications of this work.

These data undermine a scanning interpretation of fixedset memory search. The similarity between the lexical decision and the memory-search set-size functions supports our hypothesis that structural factors contribute significantly to the slope of the set-size function. One reason that the scanning hypothesis has been important is that it has been a parsimonious explanation of both fixed- and varied-set results. Because our results undermine the scanning interpretation of the fixed-set procedure, this parsimony is also undermined. However, it is important to note that these data do not necessarily undermine the usual scanning interpretation of varied-set memory-search data. There is generally not a repetition confound in the variedset procedure because set size has typically been varied randomly within blocks of trials and each probe has typically been presented an equal number of times. However, it is still possible that a dilution of priming effect may contribute to the slope of the set-size function in the varied-set procedure (Stadler, 1989).

As we pointed out in the introduction, a large body of literature has been devoted to investigating and modeling the memory-search task (see Townsend \& Ashby, 1983). Most of these models have included some sort of scanning mechanism or stage; however, if one were to model the results of the experiment reported here, there might be no need for such a search component. In addition, search models typically assume that the manipulation of memory set size selectively influences only the memorycomparison stage in the memory-search model. Our data show that this assumption is not likely to be valid, so if these models are to be complete, effects such as those observed in the present experiments must be considered and accounted for.

Perhaps the most appropriate models for the data presented here are direct-access, or activation, models of 
the memory-search data (Anderson, 1973; Atkinson \& Juola, 1974; Baddeley \& Ecob, 1973; Corballis, 1975; Hockley \& Corballis, 1982). These models provide mechanisms that are sensitive to subjects' immediate experience with items presented during the experiment. These models generally rely on a strengthening process whereby an item's strength or activation in memory is increased when an item is presented. The set-size effect is thought to occur because decision processes take longer when item strength or activation is low (because it is shared among several items) as contrasted with cases in which activation is higher (because it is distributed among fewer items). Thus, variations in memory set size affect the duration of a decision stage by affecting item strength. A comparison process in which the probe is compared to several items in memory is not necessary.

Alternatively, one might continue to work with the Sternberg model and argue that memory set size might affect stages other than the comparison stage (see, e.g., Kirsner, 1972; Strayer, Wickens, \& Braune, 1987). Kirsner (1972) used a task in which subjects named the probe after presentation of the memory set as a control for perceptual and response factors in memory search. There was an effect of set size on naming time, and this suggests that processes other than a memory-comparison process were affected by memory set size. Strayer et al. (1987) measured the P300 response ${ }^{3}$ as well as overt reaction times, and found a steeper slope for overt reaction times than for P300 latencies. Since P300 reflects preresponse processing, Strayer et al. argued that response processes contributed to the set-size effect observed with overt reaction times. Like Kirsner, they attributed much of this change in slope to processes affected by set size that are separate from the comparison process. Thus, as suggested above, one might stay within the memory-scanning framework and argue that memory set size affects other processes in addition to a memory-scanning process.

Whichever explanation one favors, it is apparent that structural factors in the fixed-set procedure contribute to or produce the set-size effect. Indeed, we have shown that the inclusion of these structural effects in a lexical decision task can produce results identical to those obtained in a memory-search task. These effects, and others (e.g., Diener, 1988), challenge the traditional interpretation of memory-search data.

\section{REFERENCES}

Anderson, J. A. (1973). A theory for the recognition of items from short memorized lists. Psychological Review, 80, 417-438.

Atkinson, R. C., Juola, J. F. (1974). Search and decision processes in recognition memory. In D. H. Krantz, R. C. Atkinson, R. D. Luce, \& P. Suppes (Eds.), Contemporary developments in mathematical psychology (Vol. 1, pp. 243-293). San Francisco: Freeman.

BADDELEY, A. D., ECOB, J. R. (1973). Reaction time and short-term memory: Implications of repetition effects for the high-speed exhaustive scan hypothesis. Quarterly Journal of Experimental Psychology, 25, 229-240.
Briggs, G. E., \& JoHNSEN, A. M. (1973). On the nature of central processing in choice reactions. Memory \& Cognition, 1, 91-100.

Burrows, D., Murdock, B. B. (1969). Effects of extended practice on high-speed scanning. Journal of Experimental Psychology, 89, 231-237

Corballis, M. C. (1975). Access to memory: An analysis of recognition times. In P. M. A. Rabbitt \& S. Dornic (Eds.), Attention and performance (Vol. 5, pp. 591-612). New York: Academic Press.

DiENER, D. (1988). Absence of the set-size effect in memory-search tasks in the absence of a preprobe delay. Memory \& Cognition, 16, 367-376

Grabol, D. (1971). Searching for targets: The effects of specific practice. Perception \& Psychophysics, 10, 300-304.

Hockley, W. E., \& Corballis, M. C. (1982). Tests of serial scanning in item recognition. Canadian Journal of Psychology, 36, 189-212

Kahneman, D., \& Chajzyck, D. (1983). Tests of the automaticity of reading: Dilution of Stroop effects by color-irrelevant stimuli. Journal of Experimental Psychology: Human Perception \& Performance, 9, 497-509.

KIRBY, J. (1980). Sequential effects in choice reaction time. In A. T. Welford (Ed.), Reaction times (pp. 129-172). New York: Academic Press.

KIRSNER, K. (1972). Developmental changes in short-term recognition memory. British Journal of Psychology, 63, 109-117.

KoRNBLUM, S. (1969). Sequential determinants of information processing in serial and discrete choice reaction time. Psychological Review, 76, 113-131.

Kornblum, S. (1973). Sequential effects in choice reaction time: A tutorial review. In S. Kornblum (Ed.), Attention and performance (Vol. 4, pp. 259-288). New York: Academic Press.

Kristofferson, M. W. (1972). When item recognition and visual search functions are similar. Perception \& Psychophysics, 12, 379-384.

KuČERA, H., \& Francis, W. N. (1967). Computational analysis of present-day American English. Providence, RI: Brown University Press.

LIVELY, B. (1972). Speed/accuracy tradeoff and practice as determinants of stage durations in a memory search task. Joumal of Experimental Psychology, 96, 97-103.

LoGAN, G. D. (1978). Attention in character classification: Evidence for the automaticity of component stages. Journal of Experimental Psychology: General, 107, 32-63.

Neisser, U., Novick, R., \& LazAr, R. (1963). Searching for ten targets simultaneously. Perceptual \& Motor Skills, 17, 955-961.

RABbitT, P. M. A., \& VyAS, S. (1973). What is repeated in the repetition effect? In S. Kornblum (Ed.), Attention and performance (Vol. 4, pp. 327-342). New York: Academic Press.

Ross, J. (1970). Extended practice with a single-character classification task. Perception \& Psychophysics, 8, 276-278.

Simpson, P. J. (1972). High-speed memory scanning: Stability and generality. Journal of Experimental Psychology, 96, 239-246.

STADLER, M. A. (1989). Dilution of priming and varied-set memory search. Manuscript in preparation.

STERNBERG, S. (1966). High-speed scanning in human memory. Science, $153,652-654$

Sternberg, S. (1967). Two operations in character recognition: Some evidence from reaction-time measurements. Perception \& Psychophysics, 2, 45-53

SternberG, S. (1969). Memory scanning: Mental processes revealed by reaction time experiments. American Scientist, 57, 421-457.

Sternberg, S. (1975). Memory scanning: New findings and current controversies. Quarterly Jourmal of Experimental Psychology, 27, 1-32.

Strayer, D. L., Wickens, C. D., \& Braune, R. (1987). Adult age differences in the speed and capacity of information processing: 2 . An electrophysiological approach. Psychology \& Aging, 2, 99-110.

Townsend, J. T., AshBy, F. G. (1983). Stochastic modeling of elementary psychological processes. Cambridge: Cambridge University Press 


\section{NOTES}

1. Only the first 12 presentations were examined because this was the number of presentations of each item in Set 4 before the break between Blocks 2 and 3 . The break might have temporarily disrupted the repetition effects. In addition, the break occurred after a different number of repetitions for each set size $(48,24,16$, and 12, for Set Sizes 1-4, respectively), which further complicated examination of further presentations.

2. Take the simplest case, in which the same item is presented on the first and second presentations. Each item in the positive and nega- tive sets is presented an equal number of times in a block of $\mathbf{4 8}$ trials; the 48 trials are sampled randomly without replacement to construct the order of presentation. Given any item presented on Trial 1, the probability that the same item will appear on Trial 2 is the number of those items remaining in the pool divided by the number of items in the pool. These probabilities are: $23 / 47(.4893), 11 / 47(.2340), 7 / 47(.1489)$, and $5 / 47(.1063)$, for Set Sizes $1-4$, respectively.

3. The P300 is an event-related electrical brain potential. Electrical activity measured on the scalp shows a positive deflection $300-800 \mathrm{msec}$ after stimulus onset, reflecting a stimulus-evaluation process (Strayer et al., 1987).

(Manuscript received June 25, 1986;

revision accepted for publication April 28, 1989.) 